\title{
INFLATABLE ANTENNA MICROWAVE RADIOMETER FOR SOIL MOISTURE MEASUREMENT
}

NASA-TM-111582 ruce M. Kendall, M. C. Bailey, and Lyle C. Schroeder

formation and Electromagnetic Technology Division NASA Langley Research Center

\author{
Richard F. Harrington \\ Old Dominion University, Department of Engineering Technology
}

\section{Introduction}

Microwave measurements of soil moisture are not being obtained at the required spatial Earth resolution with current technology. The Low Earth Orbit Microwave Radiometry Workshop held in Hampton, Virginia, September 22-24, 1992 (ref. 1), identified measurements of soil moisture at a resolution of $10 \mathrm{~km}$ as the general science driver. Recently, new novel designs for lightweight reflector systems bave been developed using deployable inflatable antenna structures which could enable lightweight real-aperture radiometers. In consideration of this, a study was conducted at the NASA Langley Research Center (LaRC) to determine the feasibility of developing a microwave radiometer system using inflatable reflector antenna technology to obtain high spatial resolution radiometric measurements of soil moisture from low Earth orbit and which could be used with a small and cost effective launch vehicle. The required high resolution with reasonable swath width coupled with the L-band measurement frequency for soil moisture dictated the use of a large (30 meter class) real aperture antenna in conjunction with a pushbroom antenna beam configuration and noise-injection type radiometer designs at 1.4 and $4.3 \mathrm{GHz}$ to produce a 370 kilometer cross-track swath with a 10 kilometer resolution as shown in figure 1, that could be packaged for launch with a Titan II class vehicle. This study includes design of the inflatable structure, control analysis, structural and thermal analysis, antenna and feed design, radiometer design, payload packaging, orbital analysis, and electromagnetic losses in the thin membrane inflatable materials. The resulting system characteristics are listed in Table I. Details of these study elements are discussed in the following.

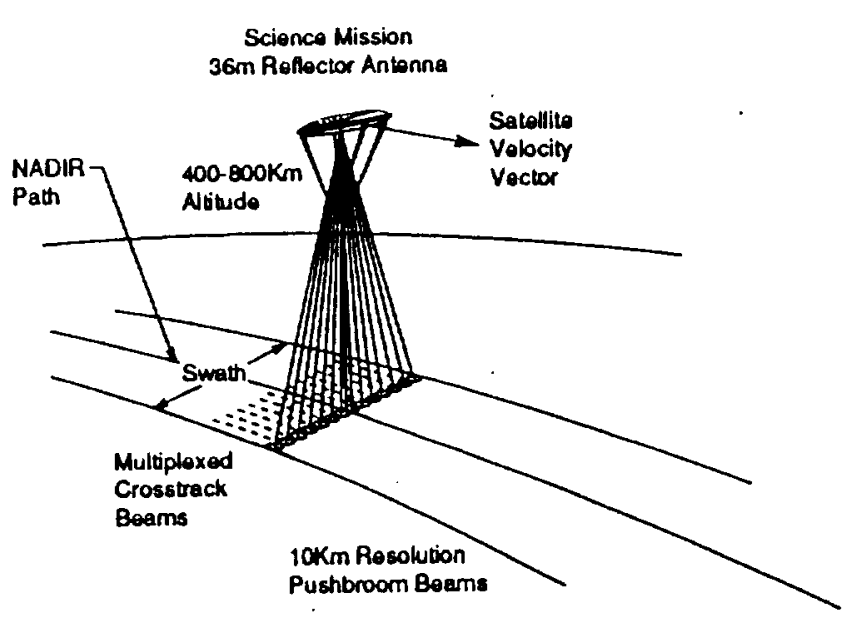

Figure 1. Inflatable antenna radiometer concept

\begin{tabular}{|l|l|}
\hline Instrument type & Noise injection Dicke radiometer \\
\hline Antenna & $36 \mathrm{~m}$ parabolic torus \\
\hline Feod & Multiplexed pushbrom \\
\hline Polarization & Circular \\
\hline Incidence angle & $0^{\circ} 1028^{\circ}$ \\
\hline Frequencies & $1.413,4.3 \mathrm{GHz}$ \\
\hline Foot-print sizes & $10,5 \mathrm{Km} @ 400 \mathrm{Km}$ alt \\
\hline Number of beams & 32,64 \\
\hline Number of radiometers & 4,12 \\
\hline Orbit altitude & $600 \pm 200 \mathrm{Km}$ \\
\hline Swath width & $370 \mathrm{Km} @ 400 \mathrm{Km}$ alt \\
\hline Instrument power & $135 \mathrm{~W}$ \\
\hline Payload weight & $2100 \mathrm{Kg}$ \\
\hline
\end{tabular}

Table 1. Inflatable antenna radiometer system characteristice

\section{Inflatable Structure Technology}

A 36-m diameter inflatable structure shaped by inflation pressure and a torus to provide an off-axis paraboloid reflector, specified by LaRC, was designed by L'Garde Inc. (ref. 2). The reflector is a metallized portion of the outer .3 mil Kapton inflatable surfaces. The total weight of the inflatable structure is 82.3 pounds and occupies 1600 in $^{3}$ of volume. The reflector inflatable requires only 6.34 pounds of gas in order to provide the correct reflector shape during a 2 year mission life. The torus is fabricated of a gelatin Kevlar material, which rigidizes by the evaporation of water in the material. Three rigidizable struts, designed to provide the correct distance between the antenna feed and the reflector surface, are fabricated of the same material as the torus. The struts are 18 in. in diameter and .015-in. thick. Since the reflector is an offaxis type, two of the struts are $71.35 \mathrm{ft}$. in length and the other strut is $76.24 \mathrm{ft}$ in length. L'Garde proposes using jack screws to adjust the distance from the reflector to the feed. The canister for housing the inflatable measures 53 $\times 42 \times 20$ inches.

\section{Radiometer Systems Technology}

\section{Reflector and Feed Design}

The configuration of the conceptional antenna design is shown in figure 2 . The reflector for the antenna consists of two aluminized "strips" on the surface of the inflatable. 
The surface is generated by rotating a parabola, with a focal length of 13 meters, about a line located a distance of 27.5 meters from the parabola vertex to produce a cylindrical surface. The projected width of the aluminized strips is 10 meters, with an offset of 2 meters. The two metallized strips are illuminated by two separate feed panels. The reflective portion of the antenna should have an rms surface roughness less than 0.02 wavelengths at the highest operating frequency. At $4.3 \mathrm{GHz}$, this surface smoothness requirement becomes 0.14 centimeters rms. The relative positional accuracy and alignment of the feed pancls with the reflective surfaces are determined by the highest operating frequency and preliminary results indicate that an accuracy of \pm 0.5 wavelengths and $\pm 0.5^{\circ}$ should be sufficient.

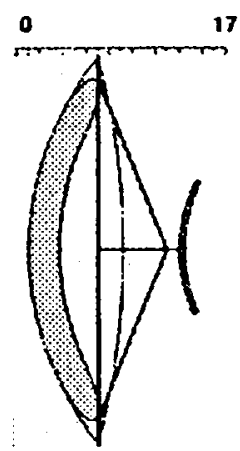

Side view

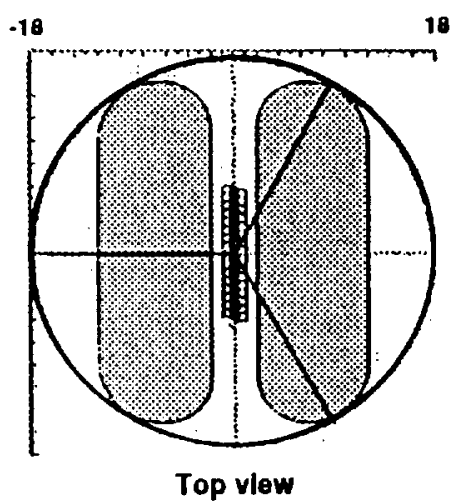

Top vlew

\section{Figure 2. Antenna sketch}

The feed panels are made up of flat square panels joined together to approximate the conical surfaces. Each of the 16 larger flat panels is a printed circuit array feed to produce one L-band (1.413 GHz) antenna beam reflected from one of the metallized strips on the antenna surface. Each of the 32 smaller flat panels is also a printed circuit array feed to produce one C-band $(4.3 \mathrm{GHz}$ ) beam. Each of the feed arrays illuminates a slightly different portion of the metallized surface and produces a beam pointing in a slightly different Earth location. The feed arrays are staggered to allow the interleaving of beams from the two separate reflective surfaces to accomplish the desired contiguous coverages.

\section{Radiometer Design}

A pushbroom radiometer design is proposed. The radiometer utilizes a balanced Dicke noise injection technique to reduce calibration periods to once per day (or once per orbit as a minimum). The design employed time multiplexing of eight antenna beams per radiometer to reduce weight and packaging. For spatial resolutions of $10 \mathrm{~km}$ and $5 \mathrm{~km}$ respectively at 1.4 and $4.3 \mathrm{GHz}$; two samples per resolution cell (Nyquist sampling criteria); and $7 \mathrm{~km}$ per sec ground track velocity; the radiometer can be time multiplexed between eight beams and still have sufficient integration time to meet the radiometric sensitivity requirement of less than $1 \mathrm{~K}$. The radiometer system consists of two major assemblies; the feed assembly and the electronics assembly. The foed assembly consists of the feed panels, the noise modules, noise source modules, and multiplexers. The electronics assembly, which is located in the spacecraft bus, contains the radiometer receivers, digital processor, data subsystem, and power supplies. The electronics assembly also provides the interface to the spacecraft's data and command systems. There are 32 antenna beams at 1.4 GHz and 64 antenna beams at $4.3 \mathrm{GHz}$. These antenna beams are arranged across track in a pushbroom configuration. The assembly also contains one data subsystem and one power subsystem along with the components for interfacing with the spacecraft systems.

\section{Electromagnetics Loss in Metallized Films \\ Because an inflatable membrane is not electromagnetically reflective by design, a metal film is added to membranes used as reflectors. If the metal film is not conductive or thick enough, the metal layer will suffer losses in the radiation gathered by the reflector. For a radiometer whose signal is composed of thermal noise, these losses cause a measurement error. For this reason, a program of analysis and measurement was undertaken to determine the loss behavior of candidate metallized film reflectors with gold, silver, and aluminum deposited at thicknesses from 100 Angstrom to 3000 Angstrom. From preliminary data, it can be concluded that, clearly, the thickest metal membranes present very low losses. Further, the gold and silver results are superior to the aluminum.}

\section{Systems Analysis}

\section{Packaging and Orbital Analysis}

The spacecraft is shown stowed in the Titan II-G beatshield in figure 3(a). A center supporn structure mounted at the vehicle interface supports all of the satellite systems. Electronics component supports and the inflatable reflector stowage container will be integral parts of the structure. The solar panels are stowed at the lower end of the structure and the feed system is attached to the top. The inflatable strut rigid deployable outriggers are folded against the center structure for launch. The hinged feeds utilize most of the available diameter of the heatshield.

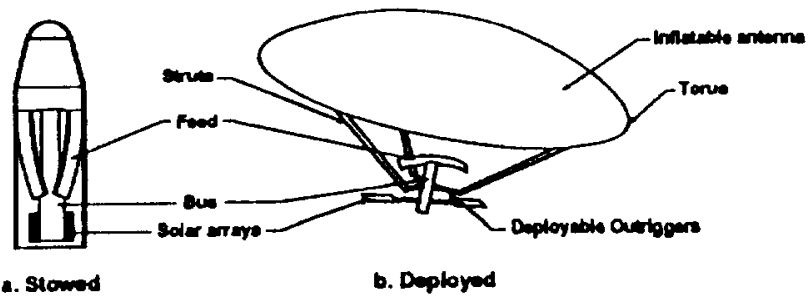

Figure 3. Spacecraft configuration 
On deployment, the bus separates from the launch vehicle and then deploy the solar panels as shown in figure 3(b). Then the feeds are deployed partially until the inflatable strut outriggers can be rotated to the final position. Next, the inflatable antenna cover is released and outriggers deployed. The antenna is then inflated and the deployment of the feeds completed. The radiometer is then ready to be oriented in the cross-track configuration. The orbital analysis used a projected area along the velocity vector of $228 \mathrm{~m}^{2}$ and spacecraft of $2090 \mathrm{~kg}$. Reboost expendables were minimized by operating as high as possible within acceptable science limits. The calculations included variations due to current models of long range solar cycle. The results showed (1) pitch variations (worst case) of $20^{\circ} @ 800 \mathrm{~km}$ and $45^{\circ} @ 700$ $\mathrm{km}$ and (best case) of $0^{\circ} @ 800 \mathrm{~km}$ and $45^{\circ} @ 450 \mathrm{~km}$; (2) required reboost fuel for 3 year lifetime (worst case) 182 $\mathrm{kg}$ for $3 / 1 / 2000$ launch (assumes reboost to $800 \mathrm{~km}$ on 1 month cycle); (3) full ground coverage time @ $800 \mathrm{~km}$ alt, 60 hours (small gaps at poles); and (4) launch vehicle proposed - Martin Marietta Titan II S-10 GEMS.

\section{Structure, Thermal, and Control Analysis}

Structural investigations of the radiometer were twophased. The first phase study showed that a three strut design was equal or superior in strut and torus bending properties, torsional rigidity, and mass; also, it will not introduce warping into the piane of the torus from strut deflections as is possible with a four strut design. The second phase of the study investigated the structural characteristics of the 37-meter cross-track inflatable antenna radiometer concept. Analytical studies with a simplified model show significant maximum thermal distortions of at least $8-10 \mathrm{~mm}$ are predicted. These results warrant further study.

An analysis of the 36-meter inflatable antenna radiometer was performed to determine the thermal behavior of an inflatable structure on-orbit. Thermal models (PATRAN, TRASYS, SINDA) were used to predict the temperature distribution of the reflector surface, canopy, torus, and struts and their variation throughout a typical orbit. Reflector temperatures range from $120^{\circ} \mathrm{F}$ at the subsolar point $10260^{\circ} \mathrm{F}$ in the Earth's shadow. Large temperature gradients occur just before and just after Earth shadow. The torus will experience similar variation in temperature as a function of orbit position. At the cold point in orbit, back side torus temperatures reach $-285^{\circ} \mathrm{F}$ while the reflector side temperatures are -30 to $-50^{\circ} \mathrm{F}$. Some rather large gradients are also present on the struts at certain points in the orbit due to shadowing from either the antenna or the spacecraft bus.

A simulation was conducted to see the effect of disturbances on the shape of the antenna's support torus. Disturbances were simulated as torques applied to the spacecraft bus near the mounting points of each strut. The magnitude of the oscillations found are small, primarily because the mass of the spacecraft bus is an order-of- magnitude larger than the mass of the antenna and so dominates the dynamic response. However, it appears that active control from the spacecraft bus can be used to increase damping in the structure's low order modes.

\section{Concluding Remarks}

The present studies indicate that, by utilizing a novel technique to obtain a large spaceborne real aperture reflector, pushbroom radiometer systems can be developed that provide passive microwave remote sensing at a spatial resolution of $10 \mathrm{~km}$ of soil moisture from space. This system could be launched with a small expendable launch vehicle, such as a Titan II. While no detailed analyses of costs were made, the systems considered herein are believed to be greater than $\$ 100$ million. The Langley Research Center is currently investigating the merits of a smaller size inflatable antenna mission on the order of 14.7 meters in terms of what science could be obtained with a payload package that uses a smaller vehicle and costs less than $\$ 100$ million. Other current investigations, which are underway at the Langley Research Center, ane the performance of advanced materials for use as radiometer antenna reflectors and research on stability of radiometer designs and components to reduce on-orbit calibration times.

\section{Acknowledgments}

The authors wish to acknowledge the other study team members for their contributions; namely, Costa Cassapakis, Koorosh Guidanean, Kevin Davey, and Art Palisoc of L'Garde, Inc. We also wish to acknowledge, at the Langley Research Center, Hans Blume and Robin Cravey of the Information and Electromagnetic Technology Division; Washito Sasamoto of the Space Systems and Concepts Division; David Cox of the Flight Dynamics and Control Division; and David Butler, Cheryl Tyler, Jill Marlowe, and William Roettker of the Aerospace Mechanical Systems Division. Additionally, Tom Campbell of the Information and Electromagnetic Technology Division at the Langley Research Center should be acknowledged for his technical leadership in initiating and supporting this study as well as Bob Freeland of the Jet Propulsion Laboratory for his support.

\section{References}

1. Low Earth Orbit Microwave Radiometry Workshop (Summary), sponsored by the Office of Space Science and Applications, NASA Headquarters, Radisson Hotel, Hampton, Virginia, September 22-24, 1992.

2. Davey, K. J.; and Palisoc, A.: Inflatable Antenna Experiment System Study. L'Garde Technical Report LTR-93-KJD-032, May 1993. 